\title{
Enhancement of Periodontal Healing by Application of a Novel Ointment Compared with Hyaluronic Acid, Histological Observation in Animal Model
}

\author{
Ghanbari Habiboallah1, Zakeri Mahdi2*, Saghravanian Nasroallah³, Zakeri Massoud², \\ Baradaran Nasseri Ehsan'2, Zareian Jahromi Mina4, Sardari Kamran ${ }^{5}$ \\ ${ }^{1}$ Department of Periodontics, School of Dentistry and Dental Research Center, Mashhad University of Medical \\ Sciences, Mashhad, Iran \\ ${ }^{2}$ School of Dentistry, Mashhad University of Medical Science, Mashhad, Iran \\ ${ }^{3}$ Department of Oral and Maxillofacial Pathology, School of Dentistry and Maxillofacial Diseases Research \\ Center, Mashhad University of Medical Sciences, Mashhad, Iran \\ ${ }^{4}$ School of Dentistry, Shahed University, Tehran, Iran \\ ${ }^{5}$ School of Veterinary Medicine, Ferdowsi University of Mashhad, Mashhad, Iran \\ Email: ${ }^{*}$ mahdi zakery@yahoo.com
}

Received 15 April 2014; revised 10 May 2014; accepted 8 June 2014

Copyright (C) 2014 by authors and Scientific Research Publishing Inc.

This work is licensed under the Creative Commons Attribution International License (CC BY).

http://creativecommons.org/licenses/by/4.0/

(c) (7) Open Access

\section{Abstract}

Objectives: The early healing of the periodontal wound in oral cavity may be critical to the treatment outcome. Thus, we made a novel ointment based on natural constituents that our recent investigation suggested a positive therapeutic effects on gingival wounds. The present study was conducted to evaluate the role of this novel biomaterial on periodontal wound healing including soft as well as hard tissue. Materials and Methods: Experimental periodontitis was induced by wire tied around the cement-enamel junction of $I_{2}, P_{1}, P_{3}$ and $M_{1}$ in six adult, healthy beagle dogs. After 3 months, mucoperiostal flap elevated and root surfaced was thoroughly scaled and planed. Randomly, three materials including our ointment with two different ratios including materials $A$ and $B$ and hyluronic acid were applied topically and then the flaps were repositioned and sutured. Three dogs at day 7 and the three remaining ones two weeks post-surgery were sacrificed and histological changes were monitored. Results: The result of this study showed that the local applications of this novel ointment promoted the healing process particularly fibroblast proliferation and collagen density remarkably in comparison with hyaluronic acid and control group. Besides, histological finding of our material revealed that in general terms had all the hallmarks of com-

${ }^{*}$ Corresponding author. 
plete healing two weeks post operatively. Conclusion: Our novel material appeared to have outstanding effect on periodontal wound healing which can improve the periodontal healing consequences and provide better strength of periodontal wound flap. From a clinical standpoint, it could play an important role for better repair in diabetic patients who frequently present difficulties in mucosal and cutanous wound healing.

\title{
Keywords
}

\author{
Anti-Inflammatory, Healing, Hyaluronic Acid, Ghee, Periodontal Flap Surgery, Animal Model
}

\section{Introduction}

Periodontal diseases are initiated by inflammatory and immunologic reactions to one or more bacterial pathogens and characterized by loss of connective tissue attachment and alveolar bone. Periodontitis has traditionally been treated by procedures such as scaling and root planning (SRP) and through the use of pharmaceuticals such as antibiotics or anti-inflammatory drugs. In most cases, after the completion of surgical periodontal procedure, the area is covered with periodontal dressing which minimizes the likelihood of post-operative infection and hemorrhage and facilitates the healing process by preventing surface trauma during mastication [1]. In general, dressing has no curative properties; however, they assist healing by protecting the tissue rather than providing "healing factor" [2]. However, the objective in wound management is promoting wound healing in the shortest time possible, with minimal pain, and discomfort to the patient.

Hyaluronic acid (hyaluronan) is a linear polysaccharide with repeating disaccharide unites glucuronic acid and $\mathrm{N}$-acetyl glucosamine without a protein core which is also an important constituent of extracellular matrix [3] [4]. Using hyaluronic acid in the form of a resorbable matrix has beneficial effect on wound healing in different regions especially periodontium following periodontal surgery [5] [6]. Some researchers reported that the local application of hyaluronic acid containing gel together with scaling and root planning has a beneficial effect in treatment of plaque-induced gingivitis [7] [8]. Conversely, other studies demonstrated that no post inflammatory tissue regeneration could be achieved by the adjunctive use of hyaluronic acid gel and SRP in the patients with chronic periodontitis [9]. In addition, hyaluronic acid-based pack did not have any significant effect on mucosal healing [10].

Besides the relatively beneficial effects of biomaterials which contain hyaluronic acid, it has several disadvantages such as complex procedure needed to be prepared, unavailability and its very high cost. Thus, we made a novel ointment entitled Curcuma longa-Ghee formulation or "CG" based on natural constituents; however, the histological finding of our recent research demonstrated that the local application of this new formulation on gingival wound sites significantly accelerated the healing process in comparison with hyluronic acid [11].

The most important components of ghee (refined animal butterfat) are saturated fatty acid and poly unsaturated fatty acid (PUFA) like linolenic acid, linoleic acid and oleic acid that in addition to their structural role can modulate cell-cell interaction and intracellular signal transduction [12]. PUFAs are also the primary precursors of many lipoic mediators like arachidonic acid with crucial function in the inflammatory process [13]. In addition, the topical application of ghee leads to faster wound closure, increase in the amount of hydroxy proline in the wound, and enhancement of wound tensile strength, and earlier epithelialization has been reported [14]. Moreover, a recent study has shown that the local application of ghee based formulation provided significantly higher collagen content and better wound contraction as well as breaking strength [15].

Curcuma longa (common tumeric) is thought to have many medicinal properties due to its antioxidant, antitumor, anti-inflammatory, and antibacterial effects. Curcumin (diferuloylmethane), a natural product obtained from the rhizomes of Curcuma longa which has been posed as an active ingredient of curcuma longa, significantly accelerated healing of wounds and enhanced wound repair in diabetic impaired healing [16]. In addition, curcumin may be useful in conditions of impaired wound healing, as it appears to induce endogenous production of TGF- $\beta 1$ in the wound which can play an important role in improvement of wound repair [17]. It has been shown that pretreatment with curcumin remarkably decreased mean wound healing time, increased synthesize of collagen and improved fibroblast densities [18]. These results agree, in part, with those obtained in a study that the authors observed faster wound closure, better maturation and cross linking of collagen in curcumin treated 
wounds [19].

The overall results of previous studies have mentioned a significant role for ghee and curcumin in accelerating wound repair. However, efforts should be directed to enhance the repair of periodontal surgical wounds and, consequently, reestablish function and esthetic in due course. Thus, on the basis of the result of our recent research, we decided to evaluate the therapeutic effects of this novel formulation compared with hyaluronic acid in inflammatory and repair stages of traumatized periodontal healing caused by experimental periodontitis following periodontal flap surgery.

\section{Materials and Methods}

\subsection{Animals}

The procedures and protocol design described here were completely in accordance with the guidelines and regulations of the Animal Research Center of Mashhad Medical Science. Six healthy 3-year-old male beagle dogs, weighing between 18 and $20 \mathrm{~kg}$ were used in this study. They had intact teeth and the clinical and radiographic survey showed no pervious periodontal disease (Figure 1). Pre-study preparation included scaling and daily plaque control to obtain periodontal health.

\subsection{Materials}

Ghee obtained from the refined sheep butterfat, was heated to $70^{\circ} \mathrm{C}$ and then mixed with the powdered rhizomes of Curcuma longa (Zingiberaceae-ginger

F. curcuma longa L. as authenticated by Dr. Davari Nejad, department of Botany, Ferdowsi University) at the same temperature under special circumstance. After cooling to $30^{\circ} \mathrm{C}-40^{\circ} \mathrm{C}$, the ointment was homogenized using a silverson homogenizer at $1500 \mathrm{rpm}$ for 40 minutes. This ointment was applied with 2 different ratio including material A (powdered rhizomes of Curcuma longa 25\% and ghee 75\%) and material B (the same ratio of powdered rhizomes of Curcuma longa and ghee). Besides, hyaluronic acid (Fibrous Fleece of hyallofill, $9.5 \mathrm{~cm}$ $\times 9.5 \mathrm{~cm}$, Convatec, US) was applied that makes a soft and sticky gel near the wound exudates due to the surgical incision.

\subsection{Surgical Protocol}

All surgical procedures were performed under general anesthesia in sterile conditions. Medetomidine hydrochloride (0.05 mg/kg, Dormitor, Orion Corporation, Espoo, Finland) was administered intramuscularly as a premedication. General anesthesia was achieved using intravenous sodium thiopental injection (25 mg/kg, Ravonal, Tanabe. Osaka, Japan), and spontaneous breathing was monitored. Routine dental infiltration anesthesia (lidocaine hydrochloride 2\%, $36 \mathrm{mg}$ in each cartridge, with epinephrine 1:80,000, Astra Pharmaceuticals, Westborough, MA) was used at the surgical sites. Experimental periodontitis was induced by a ligature (diameter 0.25 $\mathrm{mm}$, tomy International, Tokyo, Japan) tied around the cemento-enamel junction of maxillary and mandibular $\mathrm{I}_{2}$, $\mathrm{P}_{1}, \mathrm{P}_{3}$ and $\mathrm{M}_{1}$. Subsequently, no plaque control measures were performed for three months to allow accumulation of dental plaque on the wire. Acute inflammation was reduced after the ligature wire was removed, and a control plaque regimen, which consisted of a topical application of chlorhexidine (chlorhexidine gluconate 20\%, ICI Pharmaceutical Group, Wilmington, DE; $40 \mathrm{ml}$ of a $\%$ solution) three times a week for 2 weeks, was instituted. Pre-operative radiographs were exposed along with clinical assessment to confirm the presence of experimental periodontitis (Figure 2).

Following sulcular incision and elevation of buccal and lingual mucoperoistal flaps, all the granulation tissue was removed and the root surface was thoroughly scaled and planed using Gracy curettes and a chisel (HuFriedy co., Chicago, IL). On the basis of a simple randomized allocation, three materials including hyaluronic acid, material A and material B were applied topically in each two areas of six test regions and then the flaps were repositioned and sutured (Silk 0 - 3, 20 mm, Reverse cutting, Gore \& Associated Inc., Flagstaff, AZ). Subsequently, all regions including untreated wounds in the two control areas were covered with periodontal pack (Coe-PACK, GC, Japan). In order to eliminate any effect of the materials on each others, a minimum space of one tooth was considered between the above mentioned areas. An aluminum foil with 0.2 mm thickness (Toos Corporation, Mashhad, Iran) was placed on the pack and was sutured so as to prevent the removal of pack from the region of surgery. 


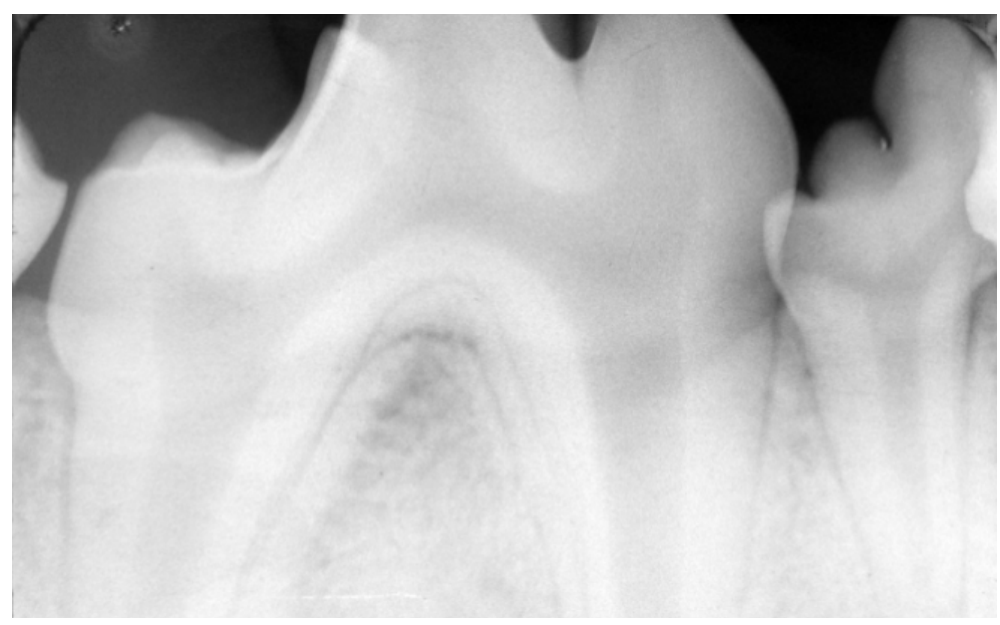

Figure 1. Radiographic examination revealed no pervious periodontal disease.

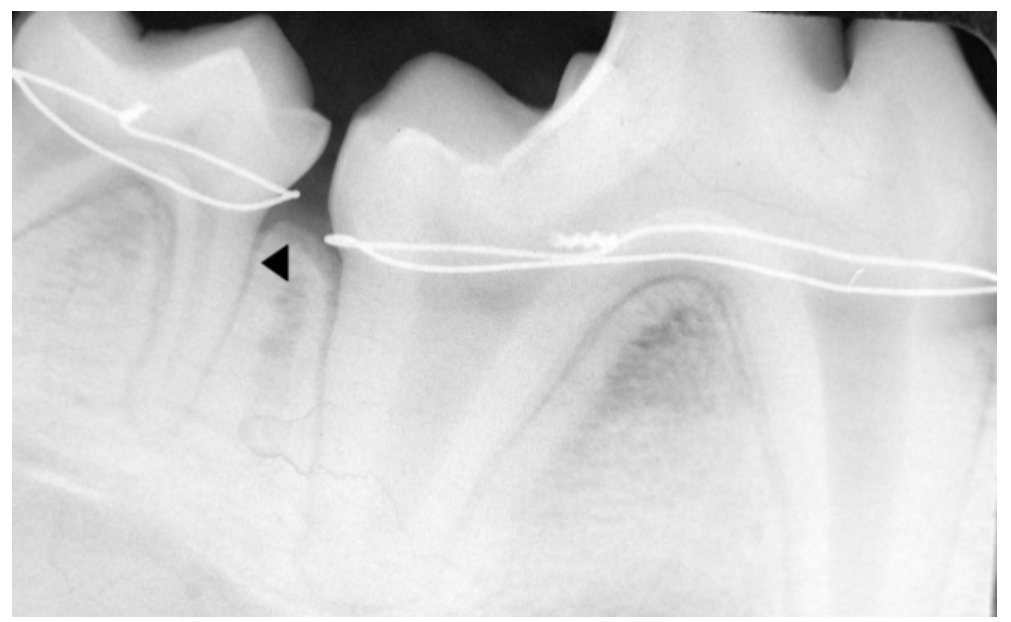

Figure 2. Radiographic survey showed crestal bone loss (Arrow head) which confirms the presence of experimental periodontitis.

\subsection{Wound Management}

A soft diet (Pedigree, Kal Kan Foods Inc., Vernon, CA) was chosen to reduce potential mechanical interference with healing during food intake. All the dogs received antibiotics (Penicillin G, 200,000 U) and an analgesic (butrophanol tartrate injection) daily for two days. Besides, plaque control was maintained daily during healing phase by topical application with chlorhexidine.

\subsection{Preparation of Histological Specimens}

Three dogs at day 7 and the three remaining ones two weeks post surgery were euthanized using an intravenous injection of concentrated of thiopental sodium. Tissue blocks including the experimental and control sites were dissected with the surrounding soft and hard tissues. The blocks were fixed in $10 \%$ buffered formalin for 3 to 5 days, decalcified in 5\% formic acid for 8 to 10 weeks, trimmed, dehydrated, and embedded in paraffin (Paraffin tissue processor HMP 110, Microm International, Heidelberg, Germany). Sections of $4 \mu \mathrm{m}$ thickness were cut in a mesio-distal plan and then were stained with hematoxylin and eosin and evaluated under light microscopy (Eclipse, E800, Nikon, Japan).

\subsection{Histologic Analysis}

The parameters used for evaluation were inflammatory cells count, necrosis, acute hemorrhage, chronic hemorr- 
hage and edema as the inflammatory parameters and neovascularization, fibroblast proliferation and collagen density as the repair parameters of the healing process [20].

Each of the parameters was scored from 0 to 3 , with $0=$ normal, $1=$ mild increase, $2=$ moderate increase, $3=$ marked increase. Sparsely scattered neutrophils, plasma cells, macrophages, eosinophils and mast cells arranged in a random fashion were considered normal. Localization of $3-10,11$ - 30, or 31 or more cells in the wound tissue per 400× magnification field was considered a mild, moderate, and marked increased, respectively.

Sparsely scattered necrotic cell debris, extravasated erythrocytes (indicative of acute hemorrhage), and haemosiderin-laden macrophages (indicative of chronic hemorrhage) were considered a mild increase for each of these parameters. Focal dense accumulations of these components in the wound tissue were considered a moderate increase. Extensive tissue necrosis, massive hemorrhage involving the surrounding tissue, and the presence of greater than 30 haemosiderin-laden macrophages per $400 \times$ magnification fields were considered a marked increase for each of these parameters, respectively.

Slight separation of cells and collagen from one another in the wound tissue by non-staining or poorly staining acellular material was considered a mild edema. Separation of $30-50 \mu \mathrm{m}$ by this acellular material was considered a moderate edema. While separation of greater than $50 \mu \mathrm{m}$ was considered a marked edema.

Fibroblasts and collagen organized in a random pattern, typical of the normal connective tissue of gingiva, were considered normal. Localization of fibroblasts and capillary buds in the wound tissue in number of $3-10$, 11 - 30, and greater than 31 per 400× magnification field were considered a mild, moderate, and a marked increase, respectively, for fibroblast and neovascularization. Scant collagen bundles slightly separating fibroblast in the wound tissues were considered a mild increase in collagen density. Somewhat dense accumulation of collagens between fibroblasts in the wound tissues was considered a moderate increase in collagen density. Extensive separation of fibroblast by abundant collagen bundles was considered a marked increase in collagen density.

\subsection{Statistical Analysis}

All samples were evaluated for one parameter before proceeding to the next which allowed for more accurate comparison of individual parameters. Mean scores were used for objective evaluation of histological changes. The data was statistically analyzed by Kruskal-Wallis test using commercial software (GraphPad Prism Version 4, GraphPad Software, San Diego, CA). Differences were considered to be staisticaly significant at $p<0.05$.

\section{Results}

\subsection{Statistical Finding}

The result of this study showed that the local application of this novel ointment promote the healing process remarkably in comparison with hyaluronic acid and control group. There was a significant difference between material B and three remaining groups in terms of inflammatory cell count and edema $(p=0.005)$, acute and chronic hemorrhage and necrosis $(p=0.006)$ at day 7. However, on comparing materials A and B with hyaluronic acid, we observed considerable difference between fibroblast proliferation $(p=0.38)$ and collagen density $(p=0.004)$ one week post-operatively (Table 1$)$.

Scores of all inflammatory and repair parameters, suggested remarkable difference at day 14 between the control group and the regions in which this three materials were applied (Table 2). Besides, there was meaningful difference between "CG" ointment and hyaluronic acid in terms of inflammatory cell count $(p=0.025)$, fibroblast proliferation $(p=0.27)$ and collagen density $(p=0.001)$ suggested the persisting inflammatory response led to relatively significant delay in the repair stage of healing two weeks after surgery in hyaluronic acid treated wounds.

\subsection{Histological Observation (One Week)}

Histological analysis of untreated wounds of control group at day 7 revealed abundant infiltration of inflammatory cells in the connective tissue supporting gingival epithelium, limited amount of vessels buds, extensive edema and poor repair parameters (Figure 3). However, we observed mild inflammatory response with scant chronic inflammatory cells among the gingival epithelial rete processes and deep in connective tissue, abundant new collagen synthesis with irregular orientation, moderate neovascularization which generally presented a relatively improved healing one week post operatively for wounds treated with material B (Figure 4). 
Table 1. Histopathologic parameters for surgical wound at day 7.

\begin{tabular}{ccccccc}
\hline & \multicolumn{5}{c}{ Mean scores } & \\
\hline & Cell Coun & Edema & Nec & Fibro & Neovas & Coll Den \\
\hline Control & $2.2(0.25)$ & $2.3(0.26)$ & $1.7(0.25)$ & $0.9(0.12)$ & $1.1(0.12)$ & $1.0(0.00)$ \\
Hyaluronic Acid & $1.8(0.31)$ & $1.6(0.18)$ & $1.5(0.18)$ & $2.1(0.29)$ & $1.5(0.18)$ & $1.9(0.22)$ \\
Material A & $2.4(0.18)$ & $2.3(0.16)$ & $1.7(0.31)$ & $2.7(0.16)$ & $2.1(0.22)$ & $2.7(0.16)$ \\
Material B & $1.1(0.12)$ & $0.9(0.12)$ & $0.5(0.18)$ & $2.8(0.12)$ & $2.0(0.26)$ & $2.8(0.12)$ \\
\hline
\end{tabular}

Number in parentheses $=$ standard error, Cell coun $=$ Cell Count Edema $=$ Edema, Nec $=$ Necrosis, Fibro $=$ Fibroblast, Neovas $=$ Neovascularization, Coll Den $=$ Collagen Density.

Table 2. Histopathologic parameters for surgical wound at day 14.

\begin{tabular}{ccccccc}
\hline & \multicolumn{7}{c}{ Mean scores } \\
& Cell Coun & Edema & Nec & Fibro & Neovas & Coll Den \\
\hline Control & $2.0(0.18)$ & $1.8(0.12)$ & $1.5(0.12)$ & $1.6(0.18)$ & $1.4(0.18)$ & $1.6(0.18)$ \\
Hyaluronic Acid & $1.5(0.18)$ & $1.1(0.12)$ & $0.9(0.22)$ & $2.3(0.26)$ & $2.0(0.18)$ & $2.1(0.12)$ \\
Material A & $1.1(0.12)$ & $0.8(0.00)$ & $0.5(0.00)$ & $3.0(0.00)$ & $1.5(0.18)$ & $3.0(0.00)$ \\
Material B & $0.6(0.00)$ & $0.7(0.16)$ & $0.5(0.18)$ & $3.0(0.00)$ & $1.5(0.18)$ & $3.0(0.00)$ \\
\hline
\end{tabular}

Number in parentheses $=$ standard error, Cell coun $=$ Cell Count, Edema $=$ Edema, Nec $=$ Necrosis, Fibro $=$ Fibroblast, Neovas $=$ Neovascularization, Coll Den = Collagen Density.

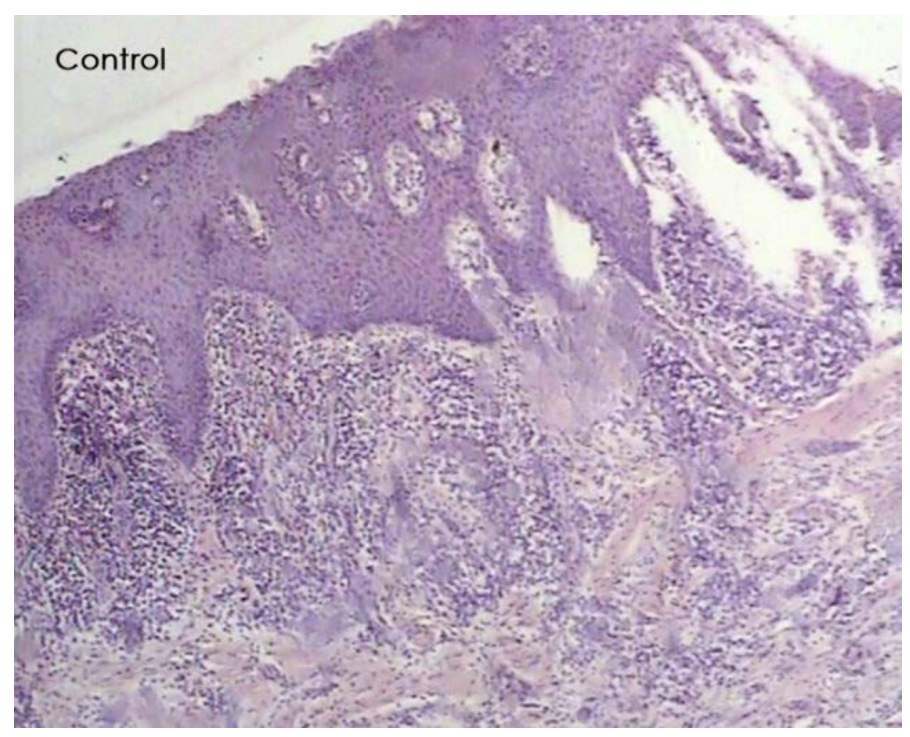

Figure 3. A histologic photomicrograph of hematoxylin and eosin stained sections of surgical wound at day 7 after the operation. (Control) Untreated wound of control group showing abundant infiltration of inflammatory cells and poor repair parameters (original magnification $4 \times$ ).

On the other hand, the amount of inflammatory parameters particularly inflammatory cell count was significantly higher $(p=0.001)$ compared with material B which probably results from the higher percentage of ghee containing prostaglandin precursors in material A. In contrast, abundant of new vessels bud and collagen synthesis directly below the gingival epithelium was apparent. Wounds treated with hyluronic acid showed moderate to severe inflammatory response, tissue disruption in periodontal ligament space, cementum resorption which consequently presented better healing compared with control group one week post operatively (Figure 5). 


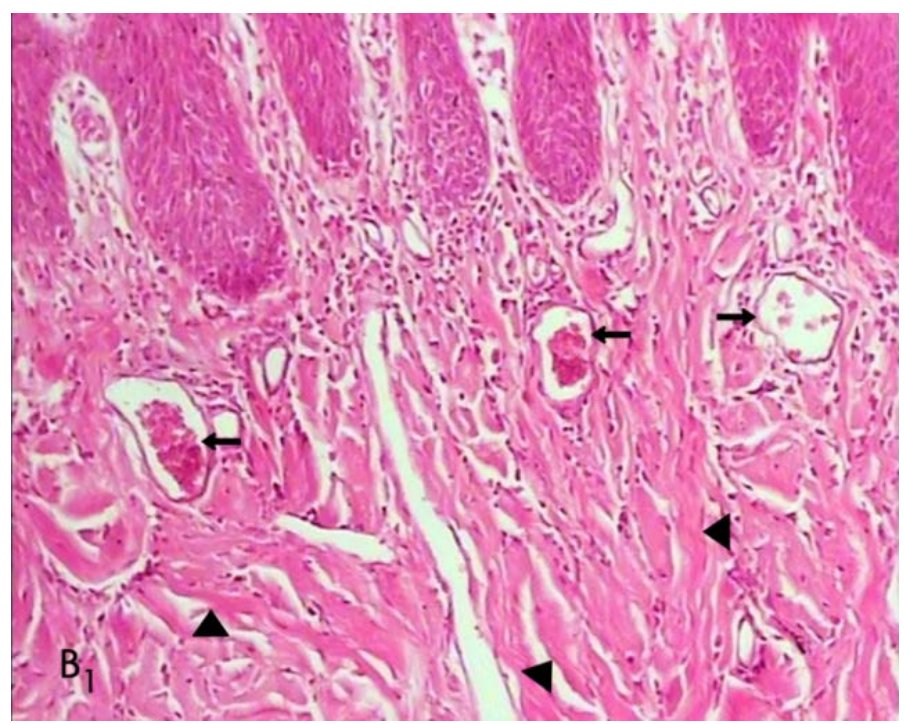

Figure 4. A histologic photomicrograph of hematoxylin and eosin stained sections of surgical wound at day 7 after the operation. $\left(B_{1}\right)$ Surgical sites treated with material B reveals scant inflammatory cells and moderate new collagen synthesis (arrow head) with irregular orientation; blood vessels (arrow) (original magnification $10 \times$ ).

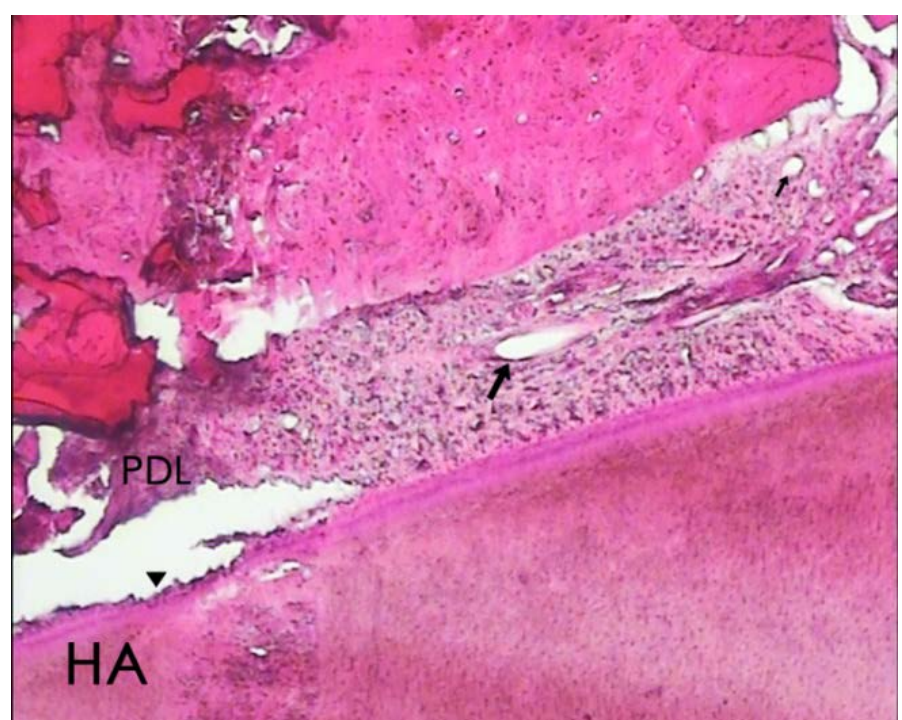

Figure 5. A histologic photomicrograph of hematoxylin and eosin stained sections of surgical wound at day 7 after the operation). (HA) wounds treated with hyaluronic acid reveals relatively severe inflammatory response particularly in periodontal ligament space (PDL) and scant new vessel blood (arrow); Cementum resorption (arrow head) (original magnification $4 \times$ ).

\subsection{Histological Observation (Two Weeks)}

In the histological survey at day 14 , in the control group, we observed moderate to severe chronic inflammatory cells accumulation particularly in lamina properia directly below the gingival epithelium and in the periodontal ligament space with extensive tissue disruption as well as edema, weak scaffold of collagen fibers which generally indicated relatively poor healing that seemed insignificant compared with day 7 . Wounds treated with hyaluronic acid presentedrelatively moderate persisting inflammatory response with highly vascularized connective tissue with extensive edema adjacent to periodontal pocket, week attachment of junctional epithelium to the root 
surface and moderate repair parameters (Figure 6). However, wounds treated with material A showed mild inflammatory response with sparsely scattered chronic inflammatory cells infiltration which appeared obviously less significant compared with day 7, dense accumulation of irregular-aligned new collagen fibers and proper attachment of connective tissue fibers throughout the root surface which indicated relatively complete healing of periodontium.

In the material B sites, we observed no signs of inflammatory response in the connective tissue and periodontal ligament, dense accumulation of relatively well-aligned new collagen fibers which attached throughout the root surface properly that in general terms has all the hallmarks of complete healing two weeks post operatively (Figure 7).

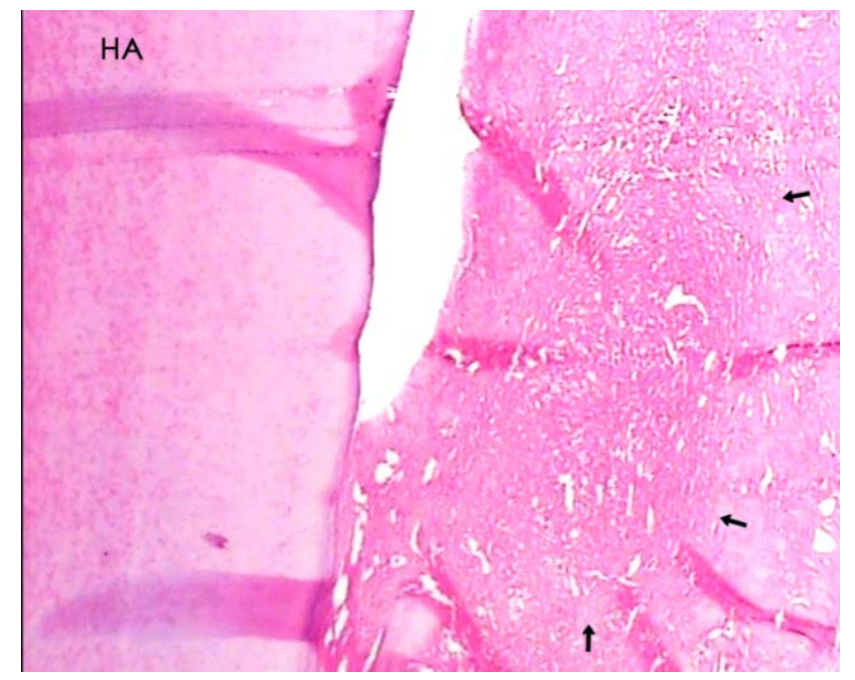

Figure 6. A histologic photomicrograph of hematoxylin and eosin stained sections of surgical wound two weeks post-operatively. (HA) Periodontal sites treated which hyaluronic acid were applied showing moderate persisting inflammatory response with extensive edema (arrows) that reveals relatively moderate healing (original magnification $4 \times$ ).

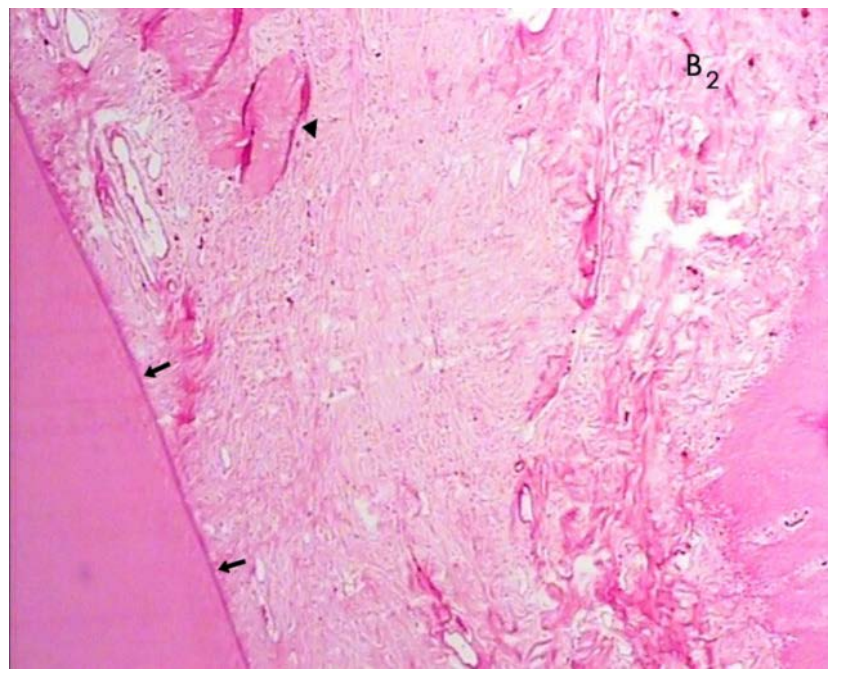

Figure 7. A histologic photomicrograph of hematoxylin and eosin stained sections of surgical wound two weeks post-operatively. $\left(\mathrm{B}_{2}\right)$ no signs of inflammatory cells in connective tissue, well-aligned new collagen fibers which attaché to the root surface thouroughly (arrows) observed in material B sites that in general terms has all the hallmarks of complete healing (original magnification $4 \times)$. 


\section{Discussion}

The phases of normal wound healing include hemostasis, inflammation, proliferation, and remodeling. Each phase of wound healing is distinct, although the wound healing process is continuous, with each phase overlapping the next. Principally, inflammation is a protective response to eliminate the initial cause of cell injury as well as necrotic cells and tissues resulting from the original insult; however, inflammation is an integral stage of wound healing process [21].

In the other hand, it is important to explore biomaterials particularly from nutritional and botanical resources to promote wound healing in the shortest time possible. However, the early strength of the periodontal wound flap and tissue function restore may be critical to the treatment outcome following surgery. Since significant finding of our pervious suggested a relevant role of this novel formulation in enhancement of gingival wounds, we designed our study to evaluate periodontal healing after local application of GC formulation in experimental periodontitis model.

Ghee comprises of saturated fatty acid and poly unsaturated fatty acid which are important arachidonic acid precursors modify the inflammatory response led to be increased [14] [20] [22]. It is interesting to note that considerable increase of inflammatory parameters in the first days of the healing process of material A compared with material B quietly agree with the fact mentioned above due to higher amount of ghee of material A.

Wounds of animals treated with curcumin have shown earlier re-epithelialization, improved neovascularization, increased migration of various cells including fibroblasts, myofibroblasts, and macrophages into the wound bed [17] as well as increased the level of TGF- $\beta 1$ in the healing tissue which, in this turn, increased granulation tissue deposition, stimulated of fibroblast production of fibronectin and collagen and its deposition in ECM which enhanced wound repair [16] [23]. These results agree with those obtained in our study that the repair parameters particularly fibroblast proliferation and collagen density remarkably increased in GC material in comparison with HA and control group one week after surgery which are the factors that are essential for wound healing; however, findings of our pervious investigation showed that gingival wounds were essentially healed at day 7 of post-operatively which confirmed this studies' results.

One of the other aims of this present work is to verify the hypothesis that the fatty acids constituents of ghee by improving the inflammatory phase and curcumin by providing better tissue formation would prove a superior treatment for healing wounds if give concomitantly preferably in one formulation. Besides,ghee undergoes deterioration gradually and the ointment formulation were evaluated in terms of free fatty acids released as it may turn rancid on storage due to presence of ghee; However, Curcumalonga has antioxidant effect [24] [25] which enhances shelf life of ghee based formulation.

In the other hand, We found a low differences between material A and material B in terms of enhancement of the surgical wound healing two weeks post-operatively, suggesting relatively similar effects of two constituents of this formulation on healing process.

In the present work, we have also observed that surgical sites treated with HA showed more intense inflammatory response compared with GC material two weeks following surgery suggesting a relatively poor basis for immigration of fibroblast and deposition of ECM lead to delay of the repair stage of healing. However, this result agrees with those we obtained in our recent research [11]. In addition, Davidson et al. suggested that the hyaluronate-treated wounds tended to accumulate collagen more slowly, which may reflect the capacity of these compounds to modify scarring process [26]; however, these results agree with finding of Min et al. showed that the application of HA may reduce scar formation whereas reduced TGF- $\beta 1$ in the healing tissue which could explained the significant lower repair parameters particularly collagen density of periodontal wounds regions which HA has been applied compared with our novel ointment at the end of second week [27].

When looking at both the inflammatory and repair stage of healing significant differences pose themselves in the early parts of the healing process. This is similar to pattern noted in other wound healing studies whereby, if a factor has a positive or negative effect on healing, its effect is most evident early during the course of healing [28] [29].

\section{Conclusions}

In summary, the GC material appeared to have outstanding effect on periodontal wound healing, which leads to improvement of therapeutic resources in the treatment of surgical wounds. From a clinical standpoint, utilization of this novel formulation could lead to improvement of periodontal treatment consequences which brings about 
better strength of periodontal wound flap and tissue function restore for the patient.

Since GC material could stimulate higher connective tissue fiber deposition, it could play an important role for better repair in diabetic patients who frequently present difficulties in mucosal and cutanous wound healing. To put in a nut shell, due to its availability and much lower cost, this new formula could be a proper substitute for hyluronic acid in improvement of surgical wound healing and it could be applied for supplementary treatment together with SRP with which clinical investigation should be done.

\section{Acknowledgements}

This experiment is supported by the Dental Research Center of the Mashhad University of Medical Sciences, Mashhad, Iran. We also wish to thanks Dr. Monavar Afzal Aghaee for her valuable input in statistical analysis of the date and Miss. Iran Momeni for helping in preparation of this novel formulation.

\section{Conflict of Interest}

The authors report no conflicts of interest.

\section{References}

[1] Sachs, H.A., Farnoush, A. and Checchi, L. (1984) Current Status of Periodontal Dressing. Journal of Periodontology, 55, 689-696. http://dx.doi.org/10.1902/jop.1984.55.12.689

[2] Smeekens, J.P., Maltha, J.C. and Renggli, H.H. (1992) Histological Evaluation of Surgically Treated Oral Tissues after Application of Photocuring Periodontal Dressing Material: An Animal Study. Journal of Clinical Periodontology, 19, 641-645. http://dx.doi.org/10.1111/j.1600-051X.1992.tb01712.x

[3] Fraser, J.R.E., Laurent, T.C. and Laurent, U.B.G. (1997) Hyaloronan: Its Nature, Distribution, Functions and Turns over. Journal of Internal Medicine, 242, 27-33. http://dx.doi.org/10.1046/j.1365-2796.1997.00170.x

[4] Le Baron, R.G., Zimmermann, D.R. and Rouslahti, E. (1992) Hyaluronate Binding Properties of Versican. Journal of Biological Chemistry, 267, 10003-10010.

[5] King, S.R., Hickerson, W.L. and Proctor, K.G. (1991) Beneficial Actions of Exogenous Hyaluronic Acid on Wound Healing. Surgery, 109, 76-86.

[6] Moseley, R., Waddington, R.J. and Embery, G. (2002) Hyaluronan and Its Potential Role in Periodontal Healing. Dental Update, 29, 144-168.

[7] Pistorius, A., Martin, M., Willershausen, B. and Rockmann, P. (2005) The Clinical Application of Hyaluronic Acid in Gingivitis Therapy. Quintessence International, 36, 531-538.

[8] Jentsch, H., Pomowski, R., Kundt, G. and Gocke, R. (2003) Treatment of Gingivitis with Hyaluronan. Journal of Clinical Periodontology, 30, 159-164. http://dx.doi.org/10.1034/j.1600-051X.2003.300203.x

[9] Xu, Y., Hofling, K., Fimmers, R., Frentzen, M. and Jervoe-Storm, P.M. (2004) Clinical and Microbiological Effect of Topical Subgingival Application of Hyaluronic Acid Gel Adjunctive to Scaling and Root Planning in the Treatment of Chronic Periodontitis. Journal of Periodontology, 75, 1114-1118. http://dx.doi.org/10.1902/jop.2004.75.8.1114

[10] Rajspaksa, S.P., Cowin, A., Adams, D. and Wormald, P.J. (2005) The Effect of a Hyaluronic Acid-Based Pack on Mucosal Healing in a Sheep Model of Sinusitis. American Journal of Rhinology, 19, 572-576.

[11] Ghanbari, H., Saghravanian, N., Zakery, M., Mahdavi Shahri, N., Zakery, M.A.S., Bardaran Nasseri, E., Zareian Jahromi, M. and Parsaei, H. (2008) Histological Evaluation of Curcuma Longa-Ghee Formulation and Hyaluronic Acid on Gingival Healing in Dog. Journal of Ethnopharmacology, 120, 335-341. http://dx.doi.org/10.1016/j.jep.2008.09.011

[12] Ruthig, D.J. and Meckling-Gill, A.K. (1999) Both (n-3) and (n-6) Fatty Acid Stimulate Wound Healing in the Rat Intestinal Epithelial Cell Line. Journal of Nutrition, 129, 1791-1798.

[13] Calder, P.C., Yaqoob, P., Thies, F., Wallace, F.A. and Miles, E.A. (2002) Fatty Acid and Lymphocyte Functions. British Journal of Nutrition, 87, 31-48. http://dx.doi.org/10.1079/BJN2001455

[14] Vure, P. and Avinash, K.D. (2006) Evaluation of Ghee Based Formulation for Wound Healing Activity. Journal of Ethnopharmacology, 107, 38-47. http://dx.doi.org/10.1016/j.jep.2006.02.006

[15] Hema, S.D., Shankar, K.M. and Bhushan, P. (2011) Wound Healing Activity of Topical Application Forms Based on Ayurveda. Evidence-Based Complementary and Alternative Medicine, 8, 1-10.

[16] Mani, H., Sidhu, G.S., Kumari, R., Gaddipati, J.P., Seth, P. and Maheshwari, R.K. (2002) Curcumin Differentially Regulates TGF- $\beta_{1}$, Its Receptors and Nitric Oxide Synthesis during Impaired Wound Healing. Biofactors, 16, 29-43. http://dx.doi.org/10.1002/biof.5520160104 
[17] Gurmel, S., Anoop, K., Deep, T., Krishna, K. and Gyanendra, K. (1998) Enhancement of Wound Healing by Curcumin in Animals. Wound Repair and Regeneration, 6, 167-177. http://dx.doi.org/10.1046/j.1524-475X.1998.60211.X

[18] Jagetia, G.C. and Rajanikant, G.K. (2004) Role of Curcumin, a Naturally Occuring Phenolic Compound of Tumeric in Accelerating the Repair of Exicision Wound in Mice Whole-Body Exposed to Various Doses of Gamma-Radiation. Journal of Surgical Research, 120, 127-138. http://dx.doi.org/10.1016/j.jss.2003.12.003

[19] Panchatcharam, M., Miriyala, S., Gayathri, V.S. and Suguna, L. (2006) Curcumin Improves Wound Healing by Modulating Collagen and Decreasing Reactive Oxygen Species. Molecular Cellular Biochemistry, 290, 87-96. http://dx.doi.org/10.1007/s11010-006-9170-2

[20] Scardino, M.E., Swaim, S.F., Strain, E.A., Hoffman, C.E., Oligive, G.K. and Hanson, R.A. (1999) The Effect of Omega-3 Fatty Acid Diet Enrichment on Wound Healing. Veterinary Dermatology, 10, 283-290. http://dx.doi.org/10.1046/j.1365-3164.1999.00148.x

[21] Martin, P., Hopkinson-Wooley, J. and McClusky, J. (1992) Growth Factor and Cutanous Wound Repair. Program of Growth factor Research, 4, 24-44.

[22] Cardose, C.R., Souza, M.A., Ferro, E.A., Favoreto Jr., S. and Pena, J.D.O. (2004) Influence of Topical Administration of n-3 and n-6 Essential and n-9 Nonessential Fatty Acid on the Healing of Cutanous Wounds. Wound Repair and Regeneration, 12, 235-243. http://dx.doi.org/10.1111/j.1067-1927.2004.012216.X

[23] Mustoe, T.A., Pierce, G.F., Morishima, C. and Devel, T.F. (1991) Growth Factors Induce Acceleration of Tissue Repair through Direct and Inductive Activities in a Rabbit Dermal Ulcer Model. Journal of Clinical Investigation, 87, 694-703. http://dx.doi.org/10.1172/JCI115048

[24] Sidhu, G.S., Mani, H., Gaddipati, J.P., Singh, A.K., Seth, P. and Banavdha, K.K. (1999) Curcumin Enhances Wound Healing in Streptozotocin Induced Diabetic Rats and Genetically Diabetic Mice. Wound Repair and Regeneration, 6, 362-374. http://dx.doi.org/10.1046/j.1524-475X.1999.00362.x

[25] Baliga, M.S., Jagetia, O.C., Rao, S.K. and Babu, K. (2003) Evaluation of Nitric Oxide Scavenging Activity of Certain Spices in Vitro: A Preliminary Study. Nahrung, 4, 261-264. http://dx.doi.org/10.1002/food.200390061

[26] Davidson, J.M., Lillian, B.N., Kenneth, N.B., Jeffrey, S.W. and Antonio, M. (1991) Hyaluronate Derivatives and Their Application to Wound Healing: Preliminary Observation. Clinical Materilas, 8, 171-177. http://dx.doi.org/10.1016/0267-6605(91)90027-D

[27] Min, H.U., Sabelman, E., Yang, C., Chang, J. and Hert, V.R. (2003) Three-Dimensional Hyaluronic Acid Graft Promotes Healing and Reduces Scar Formation in Skin Incision Wounds. Journal of Biomedical Materials Research, 678, 586-592.

[28] Lee, A.H., Swaim, S.F. and Yang, S.T. (1984) Effects of Gentamicin Solution and Cream on Healing of Open Wounds. American Journal of Veterinary Research, 45, 1487-1492.

[29] Swaim, S.F., Riddel, K.P. and McGuire, J.A. (1992) Effects of Topical Medicine on the Healing of Open Pad Wounds in Dogs. Journal of American Animal Hospital Association, 28, 499-502. 
Scientific Research Publishing (SCIRP) is one of the largest Open Access journal publishers. It is currently publishing more than 200 open access, online, peer-reviewed journals covering a wide range of academic disciplines. SCIRP serves the worldwide academic communities and contributes to the progress and application of science with its publication.

Other selected journals from SCIRP are listed as below. Submit your manuscript to us via either submit@scirp.org or Online Submission Portal.
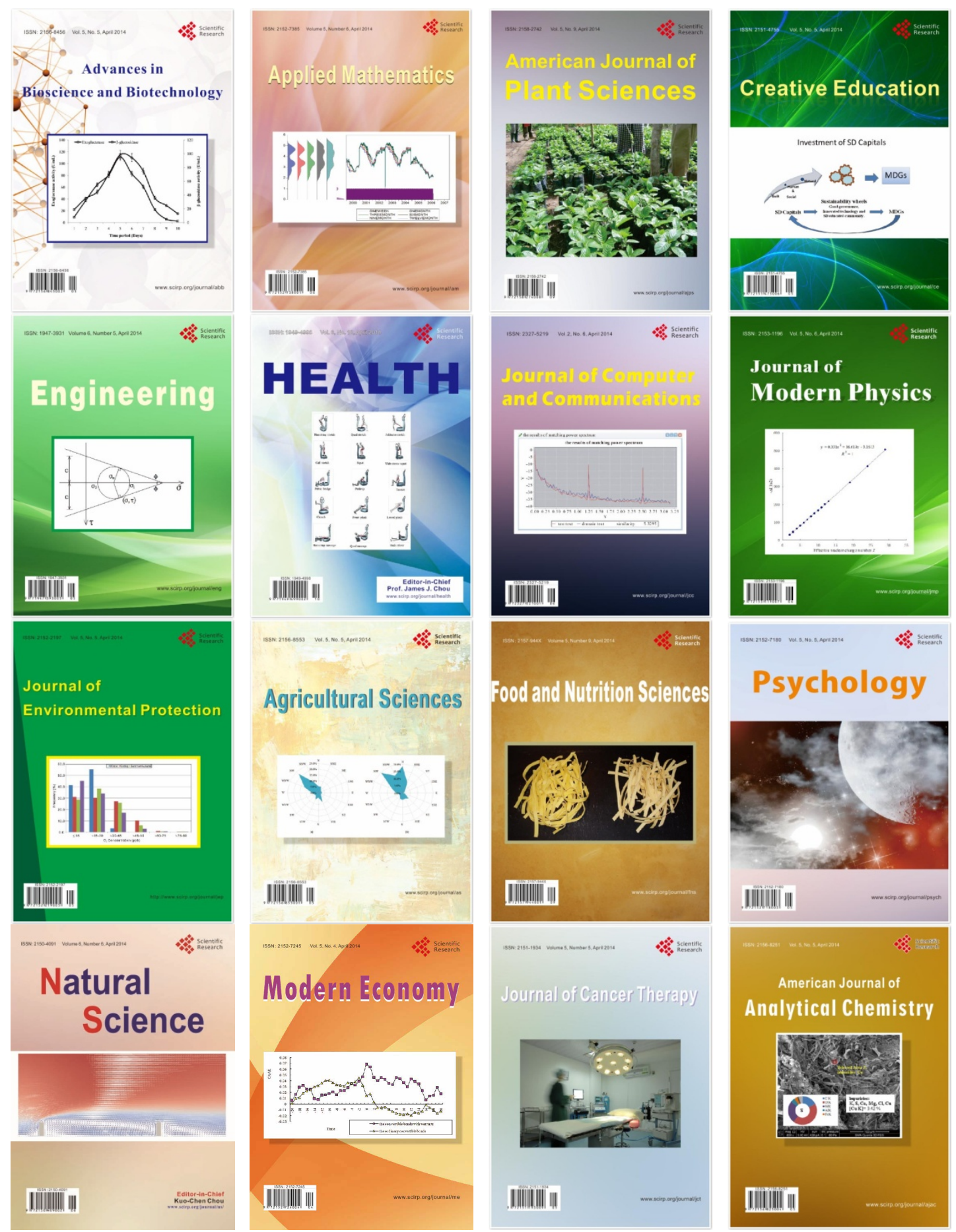